\title{
Using a clicker and a tone CS to suppress free-operant avoidance behavior
}

\author{
ALBERT E. ROBERTS \\ Catawba College, Salisbury, North Carolina
}

\begin{abstract}
Rats were trained under a free-operant avoidance schedule with R-S intervals of 15,20 , or 30 sec. Following a repeated-measures design, the rats received a 60 -sec clicking and discontinuous-tone stimulus as a preshock CS in separate training phases. Each block of CS-US sessions was preceded and followed by blocks of CS sessions without the US. The two preshock CSs produced comparable levels of suppressed avoidance behavior, as indexed by an increase in rates of unavoided shocks. The CS-US arrangements failed to produce significant differences between response-rate measures. Reliable differences in indexes of suppressed avoidance due to either the R-S interval or the kind of auditory CS were not found. However, enhancement in avoidance behavior occurred during the clicking CS in the pretest sessions.
\end{abstract}

The suppressive effects of a preshock CS on freeoperant avoidance-maintained behavior is now well documented. Conditioned avoidance suppression develops during the preshock CS; when the shock US is removed (an extinction procedure), suppressed avoidance not only disappears but is replaced by enhanced avoidance (see review by Hurwitz \& Roberts, 1977). Enhanced avoidance also develops during a CS that is given independently of both a serial compound preshock CS and the US; however, within that compound, that CS controls suppressed avoidance (Roberts \& Hurwitz, 1979).

The kind of auditory event chosen to serve as the preshock CS (a clicking or a discontinuous tone CS) seems not to have differential influence on the degree of the conditioned avoidance suppression that emerges (Roberts, 1982). One difference between these two CSs observed in the Roberts (1982) study was that the clicking CS induced markedly enhanced avoidance when given in a pretest, prior to the CS-US arrangement. This enhanced avoidance likely represented a nonassociative factor (e.g., sensitization) that arose from an interaction between the clicking event and occasional unavoided shocks. Regardless of the source of the enhancement control during the pretest, avoidance suppression developed under a subsequent CS-US arrangement.

Many studies of conditioned avoidance suppression have used shock-postponement (R-S) intervals of 15 or $20 \mathrm{sec}$ to control the baseline behavior (see Hurwitz \& Roberts, 1977). Although conditioned avoidance suppression outcome may not be bound to a narrow range of $R-S$ intervals, a specific examination of this possibility has not been undertaken. In a similar vein, the possibility that measures of avoidance suppression are affected by an in-

This research was supported by a grant from the Grants and Research Committee of Catawba College. Reprints may be obtained from the author, Department of Psychology, Catawba College, Salisbury, NC 28144 teraction between kind-of-preshock CS and R-S interval intuitively seems unlikely. However, this possibility also merits attention. The present study examined the conditioned suppression of free-operant avoidance behavior when (1) the baseline was controlled by different $R-S$ intervals and (2) a tone and a clicking stimulus served as preshock events.

\section{METHOD}

\section{Subjects}

Twelve female hooded rats purchased from Blue Spruce Farms (New York) served as subjects. The rats weighed about $190 \mathrm{~g}$ at the beginning of the experiment and were housed individually with food and water always available.

\section{Apparatus}

Three LVE operant chambers (\#143-2), measuring $25 \times 27 \times 30 \mathrm{~cm}$, were modified by placing a Chicago Miniature lamp assembly in the center of each plexiglas ceiling. A $3 \times 1 \mathrm{~cm}$ lever extended $2.5 \mathrm{~cm}$ into the center of each chamber, $4.5 \mathrm{~cm}$ above the grid floor. A deadweight of $36 \mathrm{~g}(0.36 \mathrm{~N})$ on the lever operated the microswitch. Other standard assemblies protruding into the chambers were removed. The floor grids consisted of $0.25-\mathrm{cm}$ brass rods spaced $1.3 \mathrm{~cm}$ apart parallel to the width of the lever and metal sides of the chambers. Shock was delivered to the chambers from a constant-current generator (BRS, SG901), by means of a scrambler (BRS, SC-901), and applied to the grids, lever, and metal sides of the chamber.

To serve as the clicking CS, an uncovered DPDT relay was attached to the outside of each intelligence panel and operated at 6 times/sec. To dampen the clicking sound, electrical tape was placed on the relay contacts and the electromagnet. A $2800-\mathrm{Hz}$ Mallory Sonalert also was attached to the outside of the intelligence panel (facing away from the chamber), with a 3-cm foam rubber pad over the speaker surface. The Sonalert was activated at 6 times/sec to produce the discontinuous-tone CS.

Each chamber was placed in a sound-attenuated box, with a blower fan providing ventilation and a masking noise ( $75 \mathrm{~dB}, \mathrm{SPL})$. The clicking and tone CSs added $5 \mathrm{~dB}$ and $3 \mathrm{~dB}$, respectively, to the background noise level. All programming and recording equipment were in an adjoining room.

\section{Procedure}

Under the free-operant avoidance schedule that was used, each response postponed shock for a fixed period (the R-S interval) and briefly $(0.3 \mathrm{sec})$ 
turned off the houselight. If the R-S interval elapsed, a 0.3-sec, 1-mA shock occurred every $5 \mathrm{sec}$. The rats were arranged randomly into one of three groups $(n=4)$ and given 4090 - $\min ^{1}$ and then 1096 -min avoidance-training sessions under an R-S interval of 15,20 , or $30 \mathrm{sec}$.

A block of $10 \mathrm{CS}$-pretest sessions (with the clicking stimulus or the discontinuous tone serving as the CS) followed avoidance training. The introductions of the two CSs were counterbalanced within each of the three groups. In each case, a 60-sec CS was superimposed on avoidancemaintained behavior 16 times per session on the average of once every $6 \mathrm{~min}$ (range $=2.5-12 \mathrm{~min}$ ). The first CS occurred about $7 \mathrm{~min}$ after the session began. The CS-US training phase ( 10 sessions) immediately followed. The arrangements were identical to those of the pretest phase, except that a 1-sec, 1-mA shock US followed each CS. In the CS-extinction phase (10 sessions), the shock US was not given (i.e., a procedure identical to pretest). These procedures then were replicated over three training phases with the other CS; that is, the rats initially given the tone CS received the clicking CS. Finally, the CSs were withdrawn for 10 avoidance-retraining sessions.

The experiment followed a $3 \times 14$ mixed design, with the R-S inter$\mathrm{val}$ as the between-subjects factor. The repeated-measures analysis centered on data obtained from free-operant avoidance training, the three phases under a clicking CS (CS-pretest, CS-US, and CS-extinction), the three phases under a discontinuous-tone CS (CS-pretest, CS-US, and CS-extinction), and avoidance retraining. Two measurement periods were arranged in each of the six sets of CS sessions: the 60-sec immediately preceding the CS (the baseline) and the CS periods. The number of responses and the number of unavoided shocks were recorded for each subject.

\section{RESULTS}

The mean responses/min for each subject from the final five sessions of each of the 14 measurement periods was obtained for statistical analysis. The mean number of unavoided shocks was obtained in a similar manner.

\section{Avoidance Training}

At the end of avoidance training, differences in response rates produced by the three $\mathrm{R}-\mathrm{S}$ intervals were statistically unreliable $[H(2)=2.33]$, as were differences in the rates of unavoided shocks $[H(2)=4.15]$. The responserate values and shock-rate values from each of the 14 measurement periods were pooled for separate repeatedmeasures analyses. These pooled data yielded assymetrical distributions, so Friedman and Wilcoxon tests were used for the repeated-measures analysis and individual comparisons, respectively (alpha levels $=.05$ ).

\section{CS-US Training}

Response- and shock-rate differences during the CS in the first, compared with the second, block of CS-US sessions (regardless of CS used) were not statistically significant $[T(12)=19$ and 22 , respectively]. When given first, neither the clicking nor the tone CS produced response or shock rates that were reliably different from those obtained when either CS was given second in the training sequences $[U(6,6)>12$, for all $U s]$. Without evidence for a reliable sequence effect, any liability from the pooling of data for analysis was reduced. Table 1 shows the median response and shock rates of 12 rats over the 14 measurement periods.

Response-rate measures. Differences in response rates in each of the six CS periods due to R-S interval were unreliable $[H(2)<3$, for all $H s]$. Significant differences were present between the various measurement periods $\left[F_{r}(13)=55.10 ; E_{r}^{2}=24 \%\right]$. Response rates during the clicking CS in the pretest phase were elevated significantly over the response rates in that baseline [BL; $T(12)=0]$. The response rates decreased to match BL under CS-US $[T(12)=28.5]$ but then increased over the BL of CS-extinction $[T(12)=0]$. Response rates when the clicker was given as a preshock signal were reduced significantly compared with both CS-pretest and CS-extinction [T(12) $=0$, for all $T \mathrm{~s}]$. However, the CS controlled a larger increase in response rates during the pretest than it did during extinction $[T(12)=13]$.

Under the tone CS, response rates in $\mathrm{BL}$ and $\mathrm{CS}$ periods were unreliably different during the CS-pretest and CS-US phases. ${ }^{2}$ A small but reliable increase over BL occurred during the tone in the CS-extinction phase [T(11) $=9]$.

Interestingly, all $\mathrm{BL}$ response rate values remained statistically comparable to avoidance-training levels during the various CS arrangements.

Shock-rate measures. Differences in shock rates during each of the six CS periods due to $\mathrm{R}-\mathrm{S}$ interval were not statistically significant $[H(2)<4$, for all $H$ s $]$. However, several of the differences in shock rates between measurement periods were statistically reliable $\left[F_{r}(13)=\right.$ 110.57; $E_{r}^{2}=57 \%$. Shock rates during the clicking CS

Table 1

Median Response Rates and Median Shock Rates of the 12 Rats (Based on the Final Five Sessions under Each Phase)

\begin{tabular}{|c|c|c|c|c|c|c|c|c|c|c|c|c|c|}
\hline \multirow[b]{3}{*}{$\mathrm{AV}$} & \multicolumn{6}{|c|}{ Clicking Stimulus } & \multicolumn{6}{|c|}{ Discontinuous-Tone Stimulus } & \multirow[b]{3}{*}{ AV-R } \\
\hline & \multicolumn{2}{|c|}{$\begin{array}{c}\text { CS } \\
\text { Pretest }\end{array}$} & \multicolumn{2}{|c|}{ CS-US } & \multicolumn{2}{|c|}{$\begin{array}{l}\text { CS } \\
\text { Ext }\end{array}$} & \multicolumn{2}{|c|}{$\begin{array}{c}\text { CS } \\
\text { Pretest } \\
\end{array}$} & \multicolumn{2}{|c|}{ CS-US } & \multicolumn{2}{|c|}{$\begin{array}{l}\text { CS } \\
\text { Ext }\end{array}$} & \\
\hline & BL & CS & BL & CS & BL & CS & BL & CS & BL & CS & BL & CS & \\
\hline \multicolumn{14}{|c|}{ Median Responses per Minute } \\
\hline 10.1 & 8.6 & 13.1 & 7.8 & 8.0 & 8.7 & 10.1 & 9.0 & 9.0 & 9.1 & 8.4 & 9.0 & 9.6 & 8.5 \\
\hline \multicolumn{14}{|c|}{ Median Shocks per Minute } \\
\hline .41 & .54 & .18 & .83 & 1.29 & .34 & .21 & .32 & .28 & .78 & 1.28 & .53 & .43 & .40 \\
\hline
\end{tabular}

Note-Free-operant avoidance training (AV), the three phases with the clicking CS (CS-pretest, CS-US, and CS-extinction), three identical training phases using the discontinuous tone CS, and avoidance retraining (AV-R). Baseline (BL) refers to the 60 -sec period immediately preceding CS periods. 
were lower than $\mathrm{BL}$ in the pretest, were above $\mathrm{BL}$ in CS-US, and reduced below BL under CS-extinction $[T(12)=0$, for all $T s]$. Shock rates during the tone CS were not significantly different from BL in the pretest, were increased over BL during CS-US $[T(12)=0]$, and were less than BL during CS-extinction $[T(12)=8]$. The clicker and the tone CSs produced comparable shock rates under CS-US, but under the CS-extinction, shock rates were lower during the clicking $\operatorname{CS}[T(12)=6]$.

Shock rates in BL were elevated in each of the two CS-US phases, that is, compared with avoidance training and the respective pretest periods $[T(12)=0$, for all $T$ s]; otherwise, BL shock-rate values did not differ significantly from avoidance training.

\section{Avoidance Retraining}

When the external CSs were removed for a block of avoidance-retraining sessions, both the response-rate and shock-rate values were at levels statistically comparable to those obtained at the end of avoidance training.

\section{DISCUSSION}

In the experiment described above, rats trained to postpone shock under one of three shock-postponement intervals $(15,20$, or $30 \mathrm{sec})$ received, as the CS, signaled shock with both a clicking stimulus and a discontinuous tone. CS-US training phases were preceded by a pretest and followed by an extinction phase; that is, the shock US was absent. Each preshock CS controlled suppressed avoidance behavior, as indexed by an increase, compared with BL periods, in unavoided shocks during that CS. Both the clicker and tone CS produced comparable degrees of avoidance suppression during the CS-US phases. Finally, the impaired avoidance produced by a preshock CS generalized to nonsignaled periods of the session: Shock rates during BL were increased, compared with BL values from phases that did not involve a shock US. Collectively, these results replicate earlier reports describing the effects of CS-US training on avoidance-maintained behavior (see Hurwitz \& Roberts, 1977; Roberts, 1982).

Turning to one of the purposes of this study, the suppressive effects of the auditory preshock CSs were not differentially bound to the three $\mathrm{R}-\mathrm{S}$ intervals of training. Differences in avoidance measures were not present at the end of avoidance training, and none developed during the subsequent introductions of the various CS arrangements. Similarly, evidence was not obtained that the three R-S intervals interacted with either of the two auditory CSs to differentially affect the conditioned suppression of avoidance. The use of separate R-S intervals within the same general training procedures strengthens the conclusion that the R-S interval is not a salient parameter that contributes to the effects of CS-US training.

The considered enhancement in avoidance produced by the clicking CS, but not by the tone, in a pretest confirms the earlier observation by Roberts (1982), which was based on a between-groups design. The within-subjects approach of the present study showed that avoidance enhancement to the clicking CS during the pretest was not mitigated by prior experience with CS-US. The rats receiving the clicking CS in the second CS-US block of sessions (as directed by counter balancing assignments) showed robust enhancement in the pretest period.

Most studies that present a signaled-shock arrangement do not include a CS-US replication phase, so the reintroduction of CS-US is of methodological interest. In the present experiment, the suppression of avoidance reappeared in the second occurrence of a preshock CS (albeit with a different CS). A true replication of procedure (i.e., the use of identical parameters) seems not to be present in the literature. However, Roberts \& Porter (1977) found an attenuation in avoidance impairment on a second introduction of CS-US when the duration of US was altered. This attenuation disappeared on subsequent introductions of CS-US (each with different US durations). Even so, the reap- pearance of avoidance suppression under a true replication of CS-US procedures remains to be demonstrated.

These results highlight an important issue concerning the indexing of the effects of CS-US training. The response-rate values were less a marker of suppressed avoidance behavior than were shock-rate values. For example, statistical evidence for a stronger "strength of effect" was obtained from the analysis of shock-rate values, that is, $57 \%$ compared with $24 \%$ (for response-rate values). If the analysis had focused solely on comparisons of response measures, obtained during the preshock CS, with BL, a conclusion that CS-US training had minimal effect on avoidance behavior would have been tenable. Expanding the comparisons from BL to include either of the other two CS periods (pretest or extinction) pointed to the suppressive influence of the preshock clicking CS on response rates. Even so, an advocacy for a "suppressive" effect, when the two measures obtained under the CS-US phase (BL and the preshock CS) were comparable, lacks cogency. The analysis of response-rate values obtained during the tone CS gave less compelling evidence for an acquired suppressive effect. The tone CS had no influence on response measures during the pretest and only a modest effect during CS-extinction. Only the examination of shock-rate measures identified the suppressive effects on avoidance acquired by the two preshock CSs. The reasons for the relative insensitivity of response measures are not clear, and it is likely there are several (see Hurwitz \& Herrmann, 1984). One source is the presence of multiple short-latency activations that often follow an initial shock-postponement lever activation. If the initial lever activation is induced by the delivery of an unavoided shock, the multiple activations form the well-known postshock response burst (see Hurwitz \& Roberts, 1971). Multiple activations also are not uncommon with the onset of the CS, particularly a clicking CS (see Roberts \& Hurwitz, 1970). In each case, multiple-lever activations minimally contribute to the shock-postponement requirement but do increase the recorded response frequencies. Thus, when CS-US arrangements are superimposed on avoidance-maintained behavior, unavoided shock frequencies seem the most sensitive index for assessing these arrangements.

\section{REFERENCES}

Hurwitz, H. M. B., \& Herrmann, T. F. (1984). The ineffectiveness of shock avoidance under short and long R-S intervals. Animal Learning \& Behavior, 12, 350-354.

Hurwitz, H. M. B., \& RoberTs, A. E. (1971). Conditioned suppression of an avoidance response. Journal of the Experimental Analysis of Behavior, 16, 275-281.

Hurwitz, H. M. B., \& RoberTs, A. E. (1977). Aversively controlled behavior and the analysis of conditioned suppression. In $H$. Davis \& H. M. B. Hurwitz (Eds.), Operant-Pavlovian interactions (pp. 189224). Hillsdale, NJ: Erlbaum.

RoBerTs, A. E. (1982). On nonassociative effects of auditory CSs on avoidance behavior. Bulletin of the Psychonomic Society, 19, 231-233.

RoberTs, A. E., \& HuRwitz, H. M. B. (1970). The effect of a preshock signal on a free-operant avoidance response. Journal of the Experimental Analysis of Behavior, 14, 331-340.

Roberts, A. E., \& Hurwitz, H. M. B. (1979). The conditioned suppression and enhancement of avoidance during a serial compound CS. Bulletin of the Psychonomic Society, 14, 7-10.

Roberts, A. E., \& Porter, A. G. (1977). Conditioned suppression and enhancement of avoidance in rats: The duration of US. Bulletin of the Psychonomic Society, 9, 100-102.

\section{NOTES}

1. The rats served in an observational project that was unrelated to the present study. Except for the length of the session, the training parameters of the two studies were identical.

2. Individual suppression ratios (preshock CS rate/preshock CS rate + BL rate) were obtained from response-rate values from each of the CS-US phases. The analyses of these ratios failed to yield significant group differences $[H(2)<2$, for all $H$ s].

(Manuscript received October 27, 1990.) 